Journal of Physics A 39 (2006) 14895-14910

\title{
Continuous Limit of Discrete Systems with Long-Range Interaction
}

\author{
Vasily E. Tarasov \\ Skobeltsyn Institute of Nuclear Physics, \\ Moscow State University, Moscow 119991, Russia \\ E-mail: tarasov@theory.sinp.msu.ru
}

\begin{abstract}
Discrete systems with long-range interactions are considered. Continuous medium models as continuous limit of discrete chain system are defined. Long-range interactions of chain elements that give the fractional equations for the medium model are discussed. The chain equations of motion with long-range interaction are mapped into the continuum equation with the Riesz fractional derivative. We formulate the consistent definition of continuous limit for the systems with long-range interactions. In this paper, we consider a wide class of long-range interactions that give fractional medium equations in the continuous limit. The power-law interaction is a special case of this class.
\end{abstract}

PACS: 45.05.+x; 45.50.-j; 45.10.Hj

Keywords: Discrete system, Long-range interaction, Continuous limit, Fractional equations

\section{Introduction}

Derivatives or integrals of noninteger order [1, 2, 3, 4, 5] have found many applications in recent studies in mechanics and physics [6, 7, 8, 9, 10]. Equations which involve derivatives or integrals of non-integer order are very successful in describing anomalous kinetics and transport and continuous time random walks [11, 12, 13, 14, 15. Usually, the fractional equations for dynamics or kinetics appear as some phenomenological models. Recently, a method to obtain fractional analogues of equations of motion was considered for sets of coupled particles with a long-range interaction [16, 17, 18, 19. Examples of systems with interacting oscillators, spins or waves are used for many applications in physics, chemistry, biology [20, 21, 22, 23, 24, 25, 26, 27, 28, 29, 30, 31, 32, 33, 34]. In the continuous limit, the equations of motion for discrete systems give the continuous medium equation. The procedure has already been used to derive 
fractional sine-Gordon and fractional wave Hilbert equation [16, 18], to study synchronization of coupled oscillators [17, to derive fractional Ginzburg-Landau equation [17] and for chaos in discrete nonlinear Schrödinger equation [19]. In [16, 18, 17, 19], only the power-law long-range interactions are considered. In this paper, we consider a wide class of long-range interactions that give fractional medium equations in continuous limit. The power-law interaction is a special case of this class.

Long-range interaction (LRI) has been the subject of investigations for a long time. An infinite one-dimensional Ising model with LRI was considered by Dyson [20]. The $d$-dimensional classical Heisenberg model with long-range interaction is described in [21, 22], and their quantum generalization can be found in [23, 24]. Solitons in a one-dimensional lattice with the longrange Lennard-Jones-type interaction were considered in 28]. Kinks in the Frenkel-Kontorova model with long-range interparticle interactions were studied in [29]. The properties of time periodic spatially localized solutions (breathers) on discrete chains in the presence of algebraically decaying interactions were described in [32, 33. Energy and decay properties of discrete breathers in systems with LRI have also been studied in the framework of the Klein-Gordon [27], and discrete nonlinear Schrödinger equations [30]. A main property of the dynamics described by the equation with fractional space derivatives is that the solutions have power-like tails. The lattice models with power-like long-range interactions [31, 32, 33, 35, 36, 37, 18] have similar properties. As was shown in [17, 18, 19], the analysis of the equations with fractional derivatives can provide results for the space asymptotics of their solutions.

The goal of this paper is to formulate the consistent definition of continuous limit (transform operation) for the systems with long-range interactions (LRI). This aim is realized by Propositions 1, 4, and Definitions 1, 2. The power-law LRI is considered in [16, 17, 18, 19]. The exact continuous limit results for power-law LRI were formulated in Propositions 2, 3. This operation is used to consider a wide class of long-range interactions that can be called alpha-interaction. In continuous limit, the equations of motion gives the medium equations with fractional derivatives. The power-law interaction is a special case of this class of $\alpha$-interactions. We show how the continuous limit for the systems of oscillators with long-range interaction can be described by the corresponding fractional equation.

In Sec. 2, the transform operation that maps the discrete equations into continuous medium equation is defined. In Sec. 3, the Fourier series transform of the equations of a system with long-range interaction is realized. A wide class of long-range interactions that can give the fractional equations in the continuous limit is considered. In Sec. 4, the fractional equations are obtained from three-dimensional discrete system. In Sec. 5, the linear power-law long-range interactions with positive integer and noninteger powers are considered. The correspondent continuous medium equations are discussed. In Sec. 6, the nonlinear long-range interactions for the discrete systems are used to derive the Burgers, Korteweg-de Vries, and Boussinesq equations and their fractional generalizations in the continuous limit. The conclusion is given in Sec. 7. 


\section{Transform operation}

Let us consider a one-dimensional system of interacting oscillators that are described by the equations of motion,

$$
\frac{\partial^{s} u_{n}}{\partial t^{s}}=g \hat{I}_{n}(u)+F\left(u_{n}\right)
$$

where $s=1,2$, and $u_{n}$ are displacements from the equilibrium. The terms $F\left(u_{n}\right)$ characterize an interaction of the oscillators with the external on-site force. The term $\hat{I}_{n}(u)$ is defined by

$$
\hat{I}_{n}(u) \equiv \sum_{\substack{m=-\infty \\ m \neq n}}^{+\infty} J(n, m) W\left(u_{n}, u_{m}\right)
$$

and it takes into account the interaction of the oscillators in the system.

For linear long-range interaction we have $W\left(u_{n}, u_{m}\right)=u_{n}-u_{m}$, and the interaction term (2) is

$$
\hat{I}_{n}(u) \equiv \sum_{\substack{m=-\infty \\ m \neq n}}^{+\infty} J(n, m)\left[u_{n}-u_{m}\right]
$$

In this paper, we consider a wide class of interactions (3) that create a possibility of presenting the continuous medium equations with fractional derivatives. We also discuss the term (2) with $W\left(u_{n}, u_{m}\right)=f\left(u_{n}\right)-f\left(u_{m}\right)$ as nonlinear long-range interaction. As the examples, we consider $f(u)=u^{2}$ and $f(u)=u-g u^{2}$ that gives the Burgers, Korteweg-de Vries and Boussinesq equations and their fractional generalizations in the continuous limit.

Let us define the operation, which transforms equations (1) for $u_{n}(t)$ into continuous medium equation for $u(x, t)$. We assume that $u_{n}(t)$ are Fourier coefficients of some function $\hat{u}(k, t)$. Then we define the field $\hat{u}(k, t)$ on $[-K / 2, K / 2]$ as

$$
\hat{u}(k, t)=\sum_{n=-\infty}^{+\infty} u_{n}(t) e^{-i k x_{n}}=\mathcal{F}_{\Delta}\left\{u_{n}(t)\right\},
$$

where $x_{n}=n \Delta x, \Delta x=2 \pi / K$ is distance between oscillators, and

$$
u_{n}(t)=\frac{1}{K} \int_{-K / 2}^{+K / 2} d k \hat{u}(k, t) e^{i k x_{n}}=\mathcal{F}_{\Delta}^{-1}\{\hat{u}(k, t)\}
$$

These equations are the basis for the Fourier transform, which is obtained by transforming from a discrete variable to a continuous one in the limit $\Delta x \rightarrow 0(K \rightarrow \infty)$. The Fourier transform can be derived from (4) and (5) in the limit as $\Delta x \rightarrow 0$. Replace the discrete 
$u_{n}(t)=(2 \pi / K) u\left(x_{n}, t\right)$ with continuous $u(x, t)$ while letting $x_{n}=n \Delta x=2 \pi n / K \rightarrow x$. Then change the sum to an integral, and equations (41), (5) become

$$
\begin{gathered}
\tilde{u}(k, t)=\int_{-\infty}^{+\infty} d x e^{-i k x} u(x, t)=\mathcal{F}\{u(x, t)\}, \\
u(x, t)=\frac{1}{2 \pi} \int_{-\infty}^{+\infty} d k e^{i k x} \tilde{u}(k, t)=\mathcal{F}^{-1}\{\tilde{u}(k, t)\} .
\end{gathered}
$$

Here,

$$
\tilde{u}(k, t)=\mathcal{L} \hat{u}(k, t),
$$

and $\mathcal{L}$ denotes the passage to the limit $\Delta x \rightarrow 0(K \rightarrow \infty)$. Note that $\tilde{u}(k, t)$ is a Fourier transform of the field $u(x, t)$, and $\hat{u}(k, t)$ is a Fourier series transform of $u_{n}(t)$, where we can use $u_{n}(t)=(2 \pi / K) u(n \Delta x, t)$. The function $\tilde{u}(k, t)$ can be derived from $\hat{u}(k, t)$ in the limit $\Delta x \rightarrow 0$.

The map of a discrete model into the continuous one can be defined by the transform operation.

Definition 1. Transform operation $\hat{T}$ is a combination $\hat{T}=\mathcal{F}^{-1} \mathcal{L} \mathcal{F}_{\Delta}$ of the operations:

1) The Fourier series transform:

$$
\mathcal{F}_{\Delta}: \quad u_{n}(t) \rightarrow \mathcal{F}_{\Delta}\left\{u_{n}(t)\right\}=\hat{u}(k, t) ;
$$

2) The passage to the limit $\Delta x \rightarrow 0$ :

$$
\mathcal{L}: \quad \hat{u}(k, t) \rightarrow \mathcal{L}\{\hat{u}(k, t)\}=\tilde{u}(k, t) ;
$$

3) The inverse Fourier transform:

$$
\mathcal{F}^{-1}: \quad \tilde{u}(k, t) \rightarrow \mathcal{F}^{-1}\{\tilde{u}(k, t)\}=u(x, t) .
$$

The operation $\hat{T}=\mathcal{F}^{-1} \mathcal{L} \mathcal{F}_{\Delta}$ is called a transform operation, since it performs a transform of a discrete model of coupled oscillators into the continuous medium model.

\section{From discrete to continuous equation}

Let us consider the interparticle interaction that is described by (3), where $J(n, m)$ satisfies the conditions

$$
J(n, m)=J(n-m)=J(m-n), \quad \sum_{n=1}^{\infty}|J(n)|^{2}<\infty .
$$

Note that $J(-n)=J(n)$. 
Definition 2. The interaction term (2) and (12) in the equation of motion (1) is called $\alpha$ interaction if the function

$$
\hat{J}_{\alpha}(k)=\sum_{\substack{n=-\infty \\ n \neq 0}}^{+\infty} e^{-i k n} J(n)=2 \sum_{n=1}^{\infty} J(n) \cos (k n)
$$

satisfies the condition

$$
\lim _{k \rightarrow 0} \frac{\left[\hat{J}_{\alpha}(k)-\hat{J}_{\alpha}(0)\right]}{|k|^{\alpha}}=A_{\alpha}
$$

where $\alpha>0$ and $0<\left|A_{\alpha}\right|<\infty$.

Condition (14) means that $\hat{J}_{\alpha}(k)-\hat{J}_{\alpha}(0)=O\left(|k|^{\alpha}\right)$, i.e.,

$$
\hat{J}_{\alpha}(k)-\hat{J}_{\alpha}(0)=A_{\alpha}|k|^{\alpha}+R_{\alpha}(k),
$$

for $k \rightarrow 0$, where

$$
\lim _{k \rightarrow 0} R_{\alpha}(k) /|k|^{\alpha}=0 .
$$

Examples of functions $J(n)$ for $\alpha$-interactions can be summarized in the table of the Appendix.

Proposition 1. The transform operation $\hat{T}$ maps the discrete equations of motion

$$
\frac{\partial^{s} u_{n}(t)}{\partial t^{s}}=g \sum_{\substack{m=-\infty \\ m \neq n}}^{+\infty} J(n, m)\left[u_{n}(t)-u_{m}(t)\right]+F\left(u_{n}(t)\right)
$$

with noninteger $\alpha$-interaction into the fractional continuous medium equations:

$$
\frac{\partial^{s}}{\partial t^{s}} u(x, t)-G_{\alpha} A_{\alpha} \frac{\partial^{\alpha}}{\partial|x|^{\alpha}} u(x, t)-F(u(x, t))=0,
$$

where $\partial^{\alpha} / \partial|x|^{\alpha}$ is the Riesz fractional derivative, and $G_{\alpha}=g|\Delta x|^{\alpha}$ is a finite parameter.

Proof. To derive the equation for the field $\hat{u}(k, t)$, we multiply equation (17) by $\exp (-i k n \Delta x)$, and summing over $n$ from $-\infty$ to $+\infty$. Then

$$
\sum_{n=-\infty}^{+\infty} e^{-i k n \Delta x} \frac{\partial^{s}}{\partial t^{s}} u_{n}(t)=g \sum_{n=-\infty}^{+\infty} \sum_{\substack{m=-\infty \\ m \neq n}}^{+\infty} e^{-i k n \Delta x} J(n, m)\left[u_{n}-u_{m}\right]+\sum_{n=-\infty}^{+\infty} e^{-i k n \Delta x} F\left(u_{n}\right) .
$$

The left-hand side of (19) gives

$$
\sum_{n=-\infty}^{+\infty} e^{-i k n \Delta x} \frac{\partial^{s} u_{n}(t)}{\partial t^{s}}=\frac{\partial^{s}}{\partial t^{s}} \sum_{n=-\infty}^{+\infty} e^{-i k n \Delta x} u_{n}(t)=\frac{\partial^{s} \hat{u}(k, t)}{\partial t^{s}}
$$


where $\hat{u}(k, t)$ is defined by (4). The second term of the right-hand side of (19) is

$$
\sum_{n=-\infty}^{+\infty} e^{-i k n \Delta x} F\left(u_{n}\right)=\mathcal{F}_{\Delta}\left\{F\left(u_{n}\right)\right\}
$$

The first term on the right-hand side of (19) is

$$
\begin{gathered}
\sum_{n=-\infty}^{+\infty} \sum_{\substack{m=-\infty \\
m \neq n}}^{+\infty} e^{-i k n \Delta x} J(n, m)\left[u_{n}-u_{m}\right]= \\
=\sum_{n=-\infty}^{+\infty} \sum_{\substack{m=-\infty \\
m \neq n}}^{+\infty} e^{-i k n \Delta x} J(n, m) u_{n}-\sum_{n=-\infty}^{+\infty} \sum_{\substack{m=-\infty \\
m \neq n}}^{+\infty} e^{-i k n \Delta x} J(n, m) u_{m} .
\end{gathered}
$$

Using (41) and (24), the first term in r.h.s. of (22) gives

$$
\sum_{n=-\infty}^{+\infty} \sum_{\substack{m=-\infty \\ m \neq n}}^{+\infty} e^{-i k n \Delta x} J(n, m) u_{n}=\sum_{n=-\infty}^{+\infty} e^{-i k n \Delta x} u_{n} \sum_{\substack{m^{\prime}=-\infty \\ m^{\prime} \neq 0}}^{+\infty} J\left(m^{\prime}\right)=\hat{u}(k, t) \hat{J}_{\alpha}(0)
$$

where we use (12) and $J\left(m^{\prime}+n, n\right)=J\left(m^{\prime}\right)$, and

$$
\hat{J}_{\alpha}(k \Delta x)=\sum_{\substack{n=-\infty \\ n \neq 0}}^{+\infty} e^{-i k n \Delta x} J(n)=\mathcal{F}_{\Delta}\{J(n)\}
$$

Note that

$$
\begin{gathered}
\sum_{n=-\infty}^{+\infty} \sum_{\substack{m=-\infty \\
m \neq n}}^{+\infty} e^{-i k n \Delta x} J(n, m) u_{m}=\sum_{m=-\infty}^{+\infty} u_{m} \sum_{\substack{n=-\infty \\
n \neq m}}^{+\infty} e^{-i k n \Delta x} J(n, m)= \\
=\sum_{m=-\infty}^{+\infty} u_{m} e^{-i k m \Delta x} \sum_{\substack{n^{\prime}=-\infty \\
n^{\prime} \neq 0}}^{+\infty} e^{-i k n^{\prime} \Delta x} J\left(n^{\prime}\right)=\hat{u}(k, t) \hat{J}_{\alpha}(k \Delta x),
\end{gathered}
$$

where $J\left(m, n^{\prime}+m\right)=J\left(n^{\prime}\right)$ is used.

As a result, equation (19) has the form

$$
\frac{\partial^{s} \hat{u}(k, t)}{\partial t^{s}}=g\left[\hat{J}_{\alpha}(0)-\hat{J}_{\alpha}(k \Delta x)\right] \hat{u}(k, t)+\mathcal{F}_{\Delta}\left\{F\left(u_{n}\right)\right\}
$$

where $\mathcal{F}_{\Delta}\left\{F\left(u_{n}\right)\right\}$ is an operator notation for the Fourier series transform of $F\left(u_{n}\right)$. 
The Fourier series transform $\mathcal{F}_{\Delta}$ of (17) gives (26). We will be interested in the limit $\Delta x \rightarrow 0$. Using (15), equation (26) can be written as

$$
\frac{\partial^{s}}{\partial t^{s}} \hat{u}(k, t)-G_{\alpha} \hat{\mathcal{T}}_{\alpha, \Delta}(k) \hat{u}(k, t)-\mathcal{F}_{\Delta}\left\{F\left(u_{n}(t)\right)\right\}=0,
$$

where we use finite parameter $G_{\alpha}=g|\Delta x|^{\alpha}$, and

$$
\hat{\mathcal{T}}_{\alpha, \Delta}(k)=-A_{\alpha}|k|^{\alpha}-R_{\alpha}(k \Delta x)|\Delta x|^{-\alpha} .
$$

Note that $R_{\alpha}$ satisfies the condition

$$
\lim _{\Delta x \rightarrow 0} \frac{R_{\alpha}(k \Delta x)}{|\Delta x|^{\alpha}}=0 .
$$

The expression for $\hat{\mathcal{T}}_{\alpha, \Delta}(k)$ can be considered as a Fourier transform of the operator (3)). Note that $g \rightarrow \infty$ for the limit $\Delta x \rightarrow 0$, if $G_{\alpha}$ is a finite parameter.

The passage to the limit $\Delta x \rightarrow 0$ for the third term of (27) gives

$$
\mathcal{L}: \mathcal{F}_{\Delta} F\left(u_{n}\right) \rightarrow \mathcal{L F}_{\Delta} F\left(u_{n}\right) .
$$

Then

$$
\mathcal{L F}_{\Delta}\left\{F\left(u_{n}\right)\right\}=\mathcal{F}\left\{\mathcal{L} F\left(u_{n}\right)\right\}=\mathcal{F}\left\{F\left(\mathcal{L} u_{n}\right)\right\}=\mathcal{F}\{F(u(x, t))\},
$$

where we use $\mathcal{L F}_{\Delta}=\mathcal{F} \mathcal{L}$.

As a result, equation (27) in the limit $\Delta x \rightarrow 0$ obtains

$$
\frac{\partial^{s}}{\partial t^{s}} \tilde{u}(k, t)-G_{\alpha} \hat{\mathcal{T}}_{\alpha}(k) \tilde{u}(k, t)-\mathcal{F}\{F(u(x, t))\}=0,
$$

where

$$
\tilde{u}(k, t)=\mathcal{L} \hat{u}(k, t), \quad \hat{\mathcal{T}}_{\alpha}(k)=\mathcal{L} \hat{\mathcal{T}}_{\alpha, \Delta}(k)=-A_{\alpha}|k|^{\alpha} .
$$

The inverse Fourier transform of (31) gives

$$
\frac{\partial^{s}}{\partial t^{s}} u(x, t)-G_{\alpha} \mathcal{T}_{\alpha}(x) u(x, t)-F(u(x, t))=0
$$

where $\mathcal{T}_{\alpha}(x)$ is an operator

$$
\mathcal{T}_{\alpha}(x)=\mathcal{F}^{-1}\left\{\hat{\mathcal{T}}_{\alpha}(k)\right\}=A_{\alpha} \frac{\partial^{\alpha}}{\partial|x|^{\alpha}}
$$

Here, we have used the connection between the Riesz fractional derivative and its Fourier transform [2]: $|k|^{\alpha} \longleftrightarrow-\partial^{\alpha} / \partial|x|^{\alpha}$.

As a result, we obtain continuous medium equations (18).

Examples of the interaction terms $J(n)$ that give the operators (33) in continuous medium equations are summarized in the following table. 


\begin{tabular}{|c|c|}
\hline$J(n)$ & $\mathcal{T}_{\alpha}(x)$ \\
\hline$\left(\frac{(-1)^{n} \pi^{\alpha+1}}{\alpha+1}-\frac{(-1)^{n} \pi^{1 / 2}}{(\alpha+1)|n|^{\alpha+1 / 2}} L_{1}(\alpha+3 / 2,1 / 2, \pi n)\right)$ & $-\partial^{\alpha} / \partial|x|^{\alpha}$ \\
\hline$\frac{(-1)^{n}}{n^{2}}$ & $-(1 / 2) \partial^{2} / \partial x^{2}$ \\
\hline$\frac{1}{n^{2}}$ & $-i \pi \partial / \partial x$ \\
\hline$|n|^{-(\beta+1)}, \quad(0<\beta<2, \beta \neq 1)$ & $-2 \Gamma(-\beta) \cos (\pi \beta / 2) \partial^{\beta} / \partial|x|^{\beta}$ \\
\hline$|n|^{-(\beta+1)}, \quad(\beta>2, \beta \neq 3,4, \ldots)$ & $\zeta(\beta-1) \partial^{2} / \partial x^{2}$ \\
\hline$\frac{(-1)^{n}}{\Gamma(1+\alpha / 2+n) \Gamma(1+\alpha / 2-n)} \quad(\beta>-1 / 2)$ & $-\frac{1}{\Gamma(\alpha+1)} \partial^{\alpha} / \partial|x|^{\alpha}$ \\
\hline$\frac{(-1)^{n}}{a^{2}-n^{2}}$ & $-\frac{a \pi}{2 \sin (\pi a)} \partial^{2} / \partial x^{2}$ \\
\hline$J(n)=1 / n !$ & $4 e i \partial / \partial x$ \\
\hline
\end{tabular}




\section{Fractional three-dimensional lattice equation}

The generalization for the three-dimensional case can be easy realized. Let us consider the three-dimensional lattice that is described by the equations of motion

$$
\frac{\partial^{s} u_{\mathbf{n}}}{\partial t^{s}}=g \sum_{\substack{\mathbf{m}=-\infty \\ \mathbf{m} \neq \mathbf{n}}}^{+\infty} J(\mathbf{n}, \mathbf{m})\left[u_{\mathbf{n}}-u_{\mathbf{m}}\right]+F\left(u_{\mathbf{n}}\right)
$$

where $\mathbf{n}=\left(n_{1}, n_{2}, n_{3}\right)$, and $J(\mathbf{n}, \mathbf{m})=J(\mathbf{n}-\mathbf{m})=J(\mathbf{m}-\mathbf{n})$. We suppose that $u_{\mathbf{n}}(t)$ are Fourier coefficients of the function $\hat{u}(\mathbf{k}, t)$ :

$$
\hat{u}(\mathbf{k}, t)=\sum_{\mathbf{n}=-\infty}^{+\infty} u_{\mathbf{n}}(t) e^{-i \mathbf{k r}_{\mathbf{n}}}=\mathcal{F}_{\Delta}\left\{u_{\mathbf{n}}(t)\right\},
$$

where $\mathbf{k}=\left(k_{1}, k_{2}, k_{3}\right)$, and

$$
\mathbf{r}_{\mathbf{n}}=\sum_{i=1}^{3} n_{i} \mathbf{a}_{i}
$$

Here, $\mathbf{a}_{i}$ are the translational vectors of the lattice. The continuous medium model can be derived in the limit $\left|\mathbf{a}_{i}\right| \rightarrow 0$.

To derive the equation for $\hat{u}(\mathbf{k}, t)$, we multiply (34) by $\exp \left(-i \mathbf{k r}_{\mathbf{n}}\right)$, and summing over $\mathbf{n}$. Then, we obtain

$$
\frac{\partial^{s} \hat{u}(\mathbf{k}, t)}{\partial t^{s}}=g\left[\hat{J}_{\alpha}(0)-\hat{J}_{\alpha}(\mathbf{k a})\right] \hat{u}(\mathbf{k}, t)+\mathcal{F}_{\Delta}\left\{F\left(u_{\mathbf{n}}\right)\right\}
$$

where $\mathcal{F}_{\Delta}\left\{F\left(u_{\mathbf{n}}\right)\right\}$ is an operator notation for the Fourier series transform of $F\left(u_{\mathbf{n}}\right)$, and

$$
\hat{J}_{\alpha}(\mathbf{k a})=\sum_{\mathbf{n}=-\infty}^{+\infty} e^{-i \mathbf{k} \mathbf{r}_{\mathbf{n}}} J(\mathbf{n})
$$

For the three-dimensional lattice, we define the $\alpha$-interaction with $\alpha=\left(\alpha_{1}, \alpha_{2}, \alpha_{3}\right)$, as an interaction that satisfies the conditions:

$$
\lim _{k \rightarrow 0} \frac{\left[\hat{J}_{\alpha}(\mathbf{k})-\hat{J}_{\alpha}(0)\right]}{\left|k_{i}\right|^{\alpha_{i}}}=A_{\alpha_{i}}, \quad(i=1,2,3),
$$

where $0<\left|A_{\alpha_{i}}\right|<\infty$. Conditions (38) mean that

$$
\hat{J}_{\alpha}(\mathbf{k})-\hat{J}_{\alpha}(0)=\sum_{i=1}^{3} A_{\alpha_{i}}\left|k_{i}\right|^{\alpha_{i}}+\sum_{i=1}^{3} R_{\alpha_{i}}(\mathbf{k})
$$


where

$$
\lim _{k_{i} \rightarrow 0} R_{\alpha_{i}}(\mathbf{k}) /\left|k_{i}\right|^{\alpha_{i}}=0 .
$$

In the continuous limit $\left(\left|\mathbf{a}_{i}\right| \rightarrow 0\right)$, the $\alpha$-interaction in the three-dimensional lattice gives the continuous medium equations with the derivatives $\partial^{\alpha_{1}} / \partial x^{\alpha_{1}}, \partial^{\alpha_{2}} / \partial y^{\alpha_{2}}$, and $\partial^{\alpha_{3}} / \partial z^{\alpha_{3}}$ :

$$
\frac{\partial^{s} u(\mathbf{r}, t)}{\partial t^{s}}=-g \sum_{i=1}^{3} A_{\alpha_{i}} \frac{\partial^{\alpha_{i}} u(\mathbf{r}, t)}{\partial|x|^{\alpha_{i}}}+F(u(\mathbf{r}, t)) .
$$

This equation describes multifractional properties of continuous medium.

\section{Linear power-law long-range interaction}

Let us consider the chain with linear long-range interaction that is defined by the equation of motion

$$
\frac{\partial^{s} u_{n}}{\partial t^{s}}=g \sum_{\substack{m=-\infty \\ m \neq n}}^{+\infty} J(n, m)\left[u_{n}-u_{m}\right]+F\left(u_{n}\right),
$$

where $J(n, m)=J(|n-m|)$, and

$$
J(n)=|n|^{-(\beta+1)}
$$

with positive integer number $\beta$.

Proposition 2. The power-law interaction (43) for the odd number $\beta$ is $\alpha$-interaction with $\alpha=1$ for $\beta=1$, and $\alpha=2$ for $\beta=3,5,7 \ldots$. For even numbers $\beta$, (43) is not $\alpha$-interaction. For odd number $\beta$, the transform operation $\hat{T}$ maps the equations of motion with the interaction (43) into the continuous medium equation (42) with derivatives of first order for $\beta=1$,

$$
\frac{\partial^{s}}{\partial t^{s}} u(x, t)-i G_{1} \frac{\partial}{\partial x} u(x, t)-F(u(x, t))=0,
$$

and the second order for other odd $\beta(\beta=2 m-1, m=2,3,4, \ldots)$,

$$
\frac{\partial^{s}}{\partial t^{s}} u(x, t)-G_{2} \frac{\partial^{2}}{\partial x^{2}} u(x, t)-F(u(x, t))=0,
$$

where

$$
G_{1}=\pi g \Delta x, \quad G_{2}=\frac{(-1)^{m-1}(2 \pi)^{2 m-2}}{4(2 m-2) !} B_{2 m-2} g(\Delta x)^{2}
$$

are the finite parameters. 
Proof. From Eq. (26), we get the equation for $\hat{u}(k, t)$ in the form

$$
\frac{\partial^{s} \hat{u}(k, t)}{\partial t^{s}}+g\left[\hat{J}_{\alpha}(k \Delta x)-\hat{J}_{\alpha}(0)\right] \hat{u}(k, t)-\mathcal{F}_{\Delta}\left\{F\left(u_{n}(t)\right)\right\}=0,
$$

where

$$
\hat{J}_{\alpha}(k \Delta x)=\sum_{\substack{n=-\infty \\ n \neq 0}}^{+\infty} e^{-i k n \Delta x}|n|^{-(1+\beta)}
$$

The function (48) can be represented by

$$
\hat{J}_{\alpha}(k \Delta x)=\sum_{n=1}^{+\infty} \frac{1}{n^{1+\beta}}\left(e^{-i k n \Delta x}+e^{i k n \Delta x}\right)=2 \sum_{n=1}^{+\infty} \frac{1}{n^{1+\beta}} \cos (k n \Delta x) .
$$

Then, we can use (section 5.4.2.7 in Ref. [47]) the relations

$$
\sum_{n=1}^{\infty} \frac{\cos (n k)}{n^{2 m}}=\frac{(-1)^{m-1}(2 \pi)^{2 m}}{2(2 m) !} B_{2 m}\left(\frac{k}{2 \pi}\right), \quad(0 \leq k \leq 2 \pi)
$$

where $m=1,2,3, \ldots$, and $B_{2 m}(z)$ are the Bernoulli polynomials [46], which are defined by

$$
B_{n}(k)=\sum_{m=0}^{n} C_{n}^{m} B_{m} k^{n-m}
$$

Here $B_{m}$ are the Bernoulli numbers. Note $B_{2 m-1}=0$ for $m=2,3,4 \ldots$ [46].

For $\beta=1$, we have

$$
\hat{J}_{\alpha}(k \Delta x)-\hat{J}_{\alpha}(0)=\frac{1}{2}(k \Delta x)^{2}-\pi k \Delta x \approx-\pi k \Delta x .
$$

For $\beta=2 m-1(m=2,3, \ldots)$,

$$
\hat{J}_{\alpha}(k)=\frac{(-1)^{m-1}}{(2 m) !}(2 \pi)^{2 m} B_{2 m}\left(\frac{k}{2 \pi}\right), \quad(0 \leq k \leq 2 \pi) .
$$

Then,

$$
\hat{J}_{\alpha}(k \Delta x)-\hat{J}_{\alpha}(0) \approx \frac{(-1)^{m-1}(2 \pi)^{2 m-2}}{4(2 m-2) !} B_{2 m-2}(k \Delta x)^{2} .
$$

For $\beta=0$, we have ([47], section 5.4.2.9.) the relation

$$
\sum_{n=1}^{\infty} \frac{\cos (n k)}{n}=-\ln [2 \sin (k / 2)]
$$


Then, the limit $\Delta x \rightarrow 0$ gives

$$
\hat{J}_{\alpha}(k \Delta x) \approx-\ln (k \Delta x) \rightarrow \infty .
$$

For even numbers $\beta$,

$$
\left|\hat{J}_{\alpha}(k \Delta x)-\hat{J}_{\alpha}(0)\right| /|k \Delta x|^{\beta} \rightarrow \infty
$$

since the expression has the logarithmic poles.

The transition to the limit $\Delta x \rightarrow 0$ in Eq. (47) with $\beta=1$ gives

$$
\frac{\partial^{s} \tilde{u}(k, t)}{\partial t^{s}}-G_{1} k \tilde{u}(k, t)-\mathcal{F}\{F(u(x, t))\}=0,
$$

where $G_{1}=\pi g \Delta x$ is a finite parameter. The inverse Fourier transform of (158) leads to the continuous medium equation (44) with coordinate derivative of first order. For $s=1$, this equation can be considered as the nonlinear Schrödinger equation.

The limit $\Delta x \rightarrow 0$ in Eq. (47) with $\beta=2 m-1(m=2,3, \ldots)$ gives

$$
\frac{\partial^{s} \tilde{u}(k, t)}{\partial t^{s}}+G_{2} k^{2} \tilde{u}(k, t)-\mathcal{F}\{F(u(x, t))\}=0,
$$

where $G_{2}$ is a finite parameter (46). The inverse Fourier transform of (59) leads to the partial differential equation (45) of second order. For $s=2$, this equation can be considered as a nonlinear diffusion equation, and for $s=1$ as a nonlinear wave equation.

Proposition 3. The power-law interaction (43) with noninteger $\beta$ is $\alpha$-interaction with $\alpha=\beta$ for $0<\beta<2$, and $\alpha=2$ for $\beta>2$. For $0<\beta<2(\beta \neq 1)$, the transform operation $\hat{T}$ maps the discrete equations with the interaction 43 into the continuous medium equation with fractional Riesz derivatives of order $\alpha$ :

$$
\frac{\partial^{s}}{\partial t^{s}} u(x, t)-G_{\alpha} A_{\alpha} \frac{\partial^{\alpha}}{\partial|x|^{\alpha}} u(x, t)=F(u(x, t)), \quad 0<\alpha<2, \quad(\alpha \neq 1) .
$$

For $\alpha>2(\alpha \neq 3,4,5, \ldots)$, the continuous medium equation has the coordinate derivatives of second order

$$
\frac{\partial^{s}}{\partial t^{s}} u(x, t)+G_{\alpha} \zeta(\alpha-1) \frac{\partial^{2}}{\partial|x|^{2}} u(x, t)=F(u(x, t)), \quad \alpha>2, \quad(\alpha \neq 3,4, \ldots) .
$$

Proof. For fractional positive $\alpha$, the function (48) can be represented by

$$
\hat{J}_{\alpha}(k \Delta x)=\sum_{n=1}^{+\infty} \frac{1}{n^{1+\alpha}}\left(e^{-i k n \Delta x}+e^{i k n \Delta x}\right)=L i_{1+\alpha}\left(e^{i k \Delta x}\right)+L i_{1+\alpha}\left(e^{-i k \Delta x}\right),
$$


where $L i_{\beta}(z)$ is a polylogarithm function. Using the series representation of the polylogarithm [45]:

$$
L i_{\beta}\left(e^{z}\right)=\Gamma(1-\beta)(-z)^{\beta-1}+\sum_{n=0}^{\infty} \frac{\zeta(\beta-n)}{n !} z^{n}, \quad|z|<2 \pi, \quad \beta \neq 1,2,3 \ldots,
$$

we obtain

$$
\hat{J}_{\alpha}(k \Delta x)=A_{\alpha}|\Delta x|^{\alpha}|k|^{\alpha}+2 \sum_{n=0}^{\infty} \frac{\zeta(1+\alpha-2 n)}{(2 n) !}(\Delta x)^{2 n}\left(-k^{2}\right)^{n}, \quad \alpha \neq 0,1,2,3 \ldots
$$

where $\zeta(z)$ is the Riemann zeta-function, $|k \Delta x|<2 \pi$, and

$$
A_{\alpha}=2 \Gamma(-\alpha) \cos \left(\frac{\pi \alpha}{2}\right)
$$

From (64), we have

$$
J_{\alpha}(0)=2 \zeta(1+\alpha)
$$

Then

$$
\hat{J}_{\alpha}(k \Delta x)-\hat{J}_{\alpha}(0)=A_{\alpha}|\Delta x|^{\alpha}|k|^{\alpha}+2 \sum_{n=1}^{\infty} \frac{\zeta(1+\alpha-2 n)}{(2 n) !}(\Delta x)^{2 n}\left(-k^{2}\right)^{n},
$$

where $\alpha \neq 0,1,2,3 \ldots$, and $|k \Delta x|<2 \pi$.

Substitution of (66) into (47) gives

$$
\begin{gathered}
\frac{\partial^{s} \hat{u}(k, t)}{\partial t^{s}}+g A_{\alpha}|\Delta x|^{\alpha}|k|^{\alpha} \hat{u}(k, t)+ \\
+2 g \sum_{n=1}^{\infty} \frac{\zeta(\alpha+1-2 n)}{(2 n) !}(\Delta x)^{2 n}\left(-k^{2}\right)^{n} \hat{u}(k, t)-\mathcal{F}_{\Delta}\left\{F\left(u_{n}(t)\right)\right\}=0 .
\end{gathered}
$$

We will be interested in the limit $\Delta x \rightarrow 0$. Then, Eq. (67) can be written as

$$
\frac{\partial^{s}}{\partial t^{s}} \hat{u}(k, t)+G_{\alpha} \hat{\mathcal{T}}_{\alpha, \Delta}(k) \hat{u}(k, t)-\mathcal{F}_{\Delta}\left\{F\left(u_{n}(t)\right)\right\}=0, \quad \alpha \neq 0,1,2, \ldots
$$

where we use the finite parameter

$$
G_{\alpha}=g|\Delta x|^{\min \{\alpha ; 2\}},
$$

and

$$
\hat{\mathcal{T}}_{\alpha, \Delta}(k)= \begin{cases}A_{\alpha}|k|^{\alpha}-|\Delta x|^{2-\alpha} \zeta(\alpha-1) k^{2}, & 0<\alpha<2, \quad(\alpha \neq 1) \\ |\Delta x|^{\alpha-2} A_{\alpha}|k|^{\alpha}-\zeta(\alpha-1) k^{2}, & \alpha>2, \quad(\alpha \neq 3,4, \ldots) .\end{cases}
$$

The expression for $\hat{\mathcal{T}}_{\alpha, \Delta}(k)$ can be considered as a Fourier transform of the interaction operator (2). From (69), we see that $g \rightarrow \infty$ for the limit $\Delta x \rightarrow 0$, and finite value of $G_{\alpha}$. 
The transition to the limit $\Delta x \rightarrow 0$ in Eq. (68) gives

$$
\frac{\partial^{s}}{\partial t^{s}} \tilde{u}(k, t)+G_{\alpha} \hat{\mathcal{T}}_{\alpha}(k) \tilde{u}(k, t)-\mathcal{F}\{F(u(x, t))\}=0 \quad(\alpha \neq 0,1,2, \ldots),
$$

where

$$
\hat{\mathcal{T}}_{\alpha}(k)= \begin{cases}A_{\alpha}|k|^{\alpha}, & 0<\alpha<2, \quad \alpha \neq 1 \\ -\zeta(\alpha-1) k^{2}, & 2<\alpha, \quad \alpha \neq 3,4, \ldots\end{cases}
$$

The inverse Fourier transform to (171) is

$$
\frac{\partial^{s}}{\partial t^{s}} u(x, t)+G_{\alpha} \mathcal{T}_{\alpha}(x) u(x, t)-F(u(x, t))=0 \quad \alpha \neq 0,1,2, \ldots
$$

where

$$
\mathcal{T}_{\alpha}(x)=\mathcal{F}^{-1}\left\{\hat{\mathcal{T}}_{\alpha}(k)\right\}= \begin{cases}-A_{\alpha} \partial^{\alpha} / \partial|x|^{\alpha}, & (0<\alpha<2, \quad \alpha \neq 1) \\ \zeta(\alpha-1) \partial^{2} / \partial|x|^{2}, & (\alpha>2, \quad \alpha \neq 3,4, \ldots) .\end{cases}
$$

As the result, we obtain the continuous medium equations (60) and (61).

For $s=1$ and $F(u)=0$, Eq. (60) is the fractional kinetic equation that describes the fractional superdiffusion [12, 15, 39]. If $F(u)$ is a sum of linear and cubic terms, then Eq. (60) has the form of the fractional Ginzburg-Landau equation [40, 41, 42, 43, 44]. A remarkable property of the dynamics described by the equation with fractional space derivatives is that the solutions have power-like tails.

\section{$6 \quad$ Nonlinear long-range interaction}

Let us consider the discrete equations with nonlinear long-range interaction:

$$
\hat{I}_{n}(u)=\sum_{\substack{m=-\infty \\ m \neq n}}^{+\infty} J_{\alpha}(n, m)\left[f\left(u_{n}\right)-f\left(u_{m}\right)\right]
$$

where $f(u)$ is a nonlinear function of $u_{n}(t)$, and $J_{\alpha}(n, m)=J_{\alpha}(n-m)$ defines the $\alpha$-interaction. As an example of $J_{\alpha}(n)$, we can use

$$
J_{\alpha}(n)=\frac{(-1)^{n}}{\Gamma(1+\alpha / 2+n) \Gamma(1+\alpha / 2-n)} .
$$

For $\alpha=1,2,3,4$, the interactions with $f(u)=u^{2}$ and $f(u)=u-g u^{2}$ give the Burgers, Korteweg-de Vries and Boussinesq equations in the continuous limit. For fractional $\alpha$ in Eq. (75), we can obtain the fractional generalization of these equations. 
Proposition 4. The transform operation maps the equations of motion

$$
\frac{\partial^{s} u_{n}(t)}{\partial t^{s}}=g \sum_{\substack{m=-\infty \\ m \neq n}}^{+\infty} J_{\alpha}(n-m)\left[f\left(u_{n}\right)-f\left(u_{m}\right)\right]+F\left(u_{n}\right)
$$

where $F$ is an external on-site force, and $J_{\alpha}(n)$ defines the $\alpha$-interaction, into the continuous medium equations

$$
\frac{\partial^{s} u(x, t)}{\partial t^{s}}=G_{\alpha} A_{\alpha} \frac{\partial^{\alpha}}{\partial|x|^{\alpha}} f(u(x, t))+F(u(x, t)),
$$

where $G_{\alpha}=g|\Delta x|^{\alpha}$ is a finite parameter.

Proof. The Fourier series transform of the interaction term (74) can be represented as

$$
\begin{aligned}
& \sum_{n=-\infty}^{+\infty} e^{-i k n \Delta x} \hat{I}_{n}(u)=\sum_{n=-\infty}^{+\infty} \sum_{\substack{m=-\infty \\
m \neq n}}^{+\infty} e^{-i k n \Delta x} J(n, m)\left[f\left(u_{n}\right)-f\left(u_{m}\right)\right]= \\
= & \sum_{n=-\infty}^{+\infty} \sum_{\substack{m=-\infty \\
m \neq n}}^{+\infty} e^{-i k n \Delta x} J(n, m) f\left(u_{n}\right)-\sum_{n=-\infty}^{+\infty} \sum_{\substack{m=-\infty \\
m \neq n}}^{+\infty} e^{-i k n \Delta x} J(n, m) f\left(u_{m}\right) .
\end{aligned}
$$

For the first term on the r.h.s. of (178):

$$
\sum_{n=-\infty}^{+\infty} \sum_{\substack{m=-\infty \\ m \neq n}}^{+\infty} e^{-i k n \Delta x} J(n, m) f\left(u_{n}\right)=\sum_{n=-\infty}^{+\infty} e^{-i k n \Delta x} f\left(u_{n}\right) \sum_{\substack{m^{\prime}=-\infty \\ m^{\prime} \neq 0}}^{+\infty} J\left(m^{\prime}\right)=\mathcal{F}_{\Delta}\left\{f\left(u_{n}\right)\right\} \hat{J}_{\alpha}(0)
$$

where we use $J\left(m^{\prime}+n, n\right)=J\left(m^{\prime}\right)$.

For the second term on the r.h.s. of (78):

$$
\begin{gathered}
\sum_{n=-\infty}^{+\infty} \sum_{\substack{m=-\infty \\
m \neq n}}^{+\infty} e^{-i k n \Delta x} J(n, m) f\left(u_{m}\right)=\sum_{m=-\infty}^{+\infty} f\left(u_{m}\right) \sum_{\substack{n=-\infty \\
n \neq m}}^{+\infty} e^{-i k n \Delta x} J(n, m)= \\
=\sum_{m=-\infty}^{+\infty} f\left(u_{m}\right) e^{-i k m \Delta x} \sum_{\substack{n^{\prime}=-\infty \\
n^{\prime} \neq 0}}^{+\infty} e^{-i k n^{\prime} \Delta x} J\left(n^{\prime}\right)=\mathcal{F}_{\Delta}\left\{f\left(u_{n}\right)\right\} \hat{J}_{\alpha}(k \Delta x),
\end{gathered}
$$

where we use $J\left(m, n^{\prime}+m\right)=J\left(n^{\prime}\right)$.

As a result, we obtain

$$
\frac{\partial^{s} \hat{u}(k, t)}{\partial t^{s}}=g\left[\hat{J}_{\alpha}(0)-\hat{J}_{\alpha}(k \Delta x)\right] \mathcal{F}_{\Delta}\left\{f\left(u_{n}\right)\right\}+\mathcal{F}_{\Delta}\left\{F\left(u_{n}\right)\right\},
$$


where $\hat{u}(k, t)=\mathcal{F}_{\Delta}\left\{u_{n}(t)\right\}$, and $\hat{J}_{\alpha}(k \Delta x)=\mathcal{F}_{\Delta}\{J(n)\}$.

For the limit $\Delta x \rightarrow 0$, Eq. (81) can be written as

$$
\frac{\partial^{s}}{\partial t^{s}} \hat{u}(k, t)-G_{\alpha} \hat{\mathcal{T}}_{\alpha, \Delta}(k) \hat{u}(k, t)-\mathcal{F}_{\Delta}\left\{F\left(u_{n}(t)\right)\right\}=0,
$$

where we use finite parameter $G_{\alpha}=g|\Delta x|^{\alpha}$, and

$$
\hat{\mathcal{T}}_{\alpha, \Delta}(k)=-A_{\alpha}|k|^{\alpha}-R_{\alpha}(k \Delta x)|\Delta x|^{-\alpha} .
$$

Here, the function $R_{\alpha}$ satisfies the condition

$$
\lim _{\Delta x \rightarrow 0} \frac{R_{\alpha}(k \Delta x)}{|\Delta x|^{\alpha}}=0 .
$$

In the limit $\Delta x \rightarrow 0$, we get

$$
\frac{\partial^{s}}{\partial t^{s}} \tilde{u}(k, t)-G_{\alpha} \hat{\mathcal{T}}_{\alpha}(k) \mathcal{F}\{f(u(x, t))\}-\mathcal{F}\{F(u(x, t))\}=0,
$$

where

$$
\tilde{u}(k, t)=\mathcal{L} \hat{u}(k, t), \quad \hat{\mathcal{T}}_{\alpha}(k)=\mathcal{L} \hat{\mathcal{T}}_{\alpha, \Delta}(k)=-A_{\alpha}|k|^{\alpha} .
$$

The inverse Fourier transform of (84) gives the continuous medium equation (77).

Let us consider examples of quadratic-nonlinear long-range interactions.

1) The continuous limit of the lattice equations

$$
\frac{\partial u_{n}(t)}{\partial t}=g_{1} \sum_{\substack{m=-\infty \\ m \neq n}}^{+\infty} J_{1}(n, m)\left[u_{n}^{2}-u_{m}^{2}\right]+g_{2} \sum_{\substack{m=-\infty \\ m \neq n}}^{+\infty} J_{2}(n, m)\left[u_{n}-u_{m}\right],
$$

where $J_{i}(n)(i=1,2)$ define the $\alpha_{i}$-interactions with $\alpha_{1}=1$ and $\alpha_{2}=2$, gives the Burgers equation [48] that is a nonlinear partial differential equation of second order:

$$
\frac{\partial}{\partial t} u(x, t)+G_{1} u(x, t) \frac{\partial}{\partial x} u(x, t)-G_{2} \frac{\partial^{2}}{\partial x^{2}} u(x, t)=0 .
$$

It is used in fluid dynamics as a simplified model for turbulence, boundary layer behavior, shock wave formation, and mass transport. If we consider $J_{2}(n, m)$ with fractional $\alpha_{2}=\alpha$, then we get the fractional Burgers equation that is suggested in [49]. In general, the fractional Burgers equation is

$$
\frac{\partial}{\partial t} u(x, t)+G_{\alpha_{1}} u(x, t) \frac{\partial^{\alpha_{1}}}{\partial|x|^{\alpha_{1}}} u(x, t)-G_{\alpha_{2}} \frac{\partial^{\alpha_{2}}}{\partial x^{\alpha_{2}}} u(x, t)=0 .
$$


2) The continuous limit of the system of equations

$$
\frac{\partial u_{n}(t)}{\partial t}=g_{1} \sum_{\substack{m=-\infty \\ m \neq n}}^{+\infty} J_{1}(n, m)\left[u_{n}^{2}-u_{m}^{2}\right]+g_{3} \sum_{\substack{m=-\infty \\ m \neq n}}^{+\infty} J_{3}(n, m)\left[u_{n}-u_{m}\right],
$$

where $J_{i}(n)(i=1,3)$ define the $\alpha_{i}$-interactions with $\alpha_{1}=1$ and $\alpha_{3}=3$, gives Korteweg-de Vries $(\mathrm{KdV})$ equation

$$
\frac{\partial}{\partial t} u(x, t)-G_{1} u(x, t) \frac{\partial}{\partial x} u(x, t)+G_{3} \frac{\partial^{3}}{\partial x^{3}} u(x, t)=0 .
$$

First formulated as a part of an analysis of shallow-water waves in canals, it has subsequently been found to be involved in a wide range of physics phenomena, especially those exhibiting shock waves, travelling waves and solitons. Certain theoretical physics phenomena in the quantum mechanics domain are explained by means of a KdV model. It is used in fluid dynamics, aerodynamics, and continuum mechanics as a model for shock wave formation, solitons, turbulence, boundary layer behavior and mass transport.

If we use noninteger $\alpha_{i}$-interactions for $J_{i}(n)$, then we get the fractional generalization of $\mathrm{KdV}$ equation [50, 51]:

$$
\frac{\partial}{\partial t} u(x, t)-G_{\alpha_{1}} u(x, t) \frac{\partial^{\alpha_{1}}}{\partial x^{\alpha_{1}}} u(x, t)+G_{\alpha_{3}} \frac{\partial^{\alpha_{3}}}{\partial x^{\alpha_{3}}} u(x, t)=0 .
$$

3) The continuous limit of the equations

$$
\frac{\partial^{2} u_{n}(t)}{\partial t^{2}}=g_{2} \sum_{\substack{m=-\infty \\ m \neq n}}^{+\infty} J_{2}(n, m)\left[f\left(u_{n}\right)-f\left(u_{m}\right)\right]+g_{4} \sum_{\substack{m=-\infty \\ m \neq n}}^{+\infty} J_{4}(n, m)\left[u_{n}-u_{m}\right],
$$

where

$$
f(u)=u-g u^{2},
$$

and $J_{i}(n)$ define the $\alpha_{i}$-interactions with $\alpha_{2}=2$ and $\alpha_{4}=4$, gives the Boussinesq equation that is a nonlinear partial differential equation of forth order

$$
\frac{\partial^{2}}{\partial t^{2}} u(x, t)-G_{2} \frac{\partial^{2}}{\partial x^{2}} u(x, t)+g G_{2} \frac{\partial^{2}}{\partial x^{2}} u^{2}(x, t)+G_{4} \frac{\partial^{4}}{\partial x^{4}} u(x, t)=0 .
$$

This equation was formulated as a part of an analysis of long waves in shallow water. It was subsequently applied to problems in the percolation of water in porous subsurface strata. It also crops up in the analysis of many other physical processes. The fractional Boussinesq equation is

$$
\frac{\partial^{2}}{\partial t^{2}} u(x, t)-G_{\alpha_{2}} \frac{\partial^{\alpha_{2}}}{\partial x^{\alpha_{2}}} u(x, t)+g G_{\alpha_{2}} \frac{\partial^{\alpha_{2}}}{\partial x^{\alpha_{2}}} u^{2}(x, t)+G_{\alpha_{4}} \frac{\partial^{\alpha_{4}}}{\partial x^{\alpha_{4}}} u(x, t)=0 .
$$




\section{Conclusion}

Discrete system of long-range interacting oscillators serve as a model for numerous applications in physics, chemistry, biology, etc. Long-range interactions are important type of interactions for complex media. An interesting situation arises when we consider a wide class of $\alpha$-interactions, where $\alpha$ is noninteger. A remarkable feature of these interactions is the existence of a transform operation that replaces the set of coupled individual oscillator equations by the continuous medium equation with the space derivative of noninteger order $\alpha$. Such a transform operation is an approximation that appears in the continuous limit. This limit allows us to consider different models in unified way by applying tools of fractional calculus.

We can assume that an asymmetric interaction term $(J(n-m) \neq J(|n-m|))$ leads to other forms of the fractional derivative [2].

Note that a fractional derivative can be results from a fractional difference as interaction term, just as an nth order difference leads to an nth derivative [2]. It follows from the representation of the Riesz fractional derivative by Grunwald-Letnikov fractional derivative:

$$
\frac{\partial^{\alpha}}{\partial|x|^{\alpha}} u(x, t) \simeq-\frac{1}{2 \cos (\pi \alpha / 2)} \frac{1}{h^{\alpha}} \sum_{n=0}^{\infty} \frac{(-1)^{n} \Gamma(\alpha+1)}{\Gamma(n+1) \Gamma(\alpha-n+1)}[u(x-n h, t)+u(x+n h, t)],
$$

where $h=\Delta x$ is the discretization parameter.

A similar approach to fractional dynamics in the context of the diffusion equation was developed in the papers [52, 53]. In those papers, a continuum limit of (non-interacting) random particle motions leads to a fractional equation.

\section{References}

[1] K.B. Oldham, J. Spanier, The Fractional Calculus (Academic Press, New York, 1974)

[2] S.G. Samko, A.A. Kilbas, O.I. Marichev, Fractional Integrals and Derivatives Theory and Applications (Gordon and Breach, New York, 1993)

[3] K.S. Miller, B. Ross, An Introduction to the Fractional Calculus and Fractional Differential Equations (Wiley, New York, 1993)

[4] I. Podlubny, Fractional Differential Equations (Academic Press, New York, 1999)

[5] A.A. Kilbas, H.M. Srivastava, J.J. Trujillo, Theory and Applications of Fractional Differential Equations (Elsevier, Amsterdam, 2006)

[6] G.M. Zaslavsky, Hamiltonian Chaos and Fractional Dynamics (Oxford University Press, Oxford, 2005)

[7] G.M. Zaslavsky, "Chaos, fractional kinetics, and anomalous transport" Phys. Rep. 371 (2002) 461-580.

[8] F. Mainardi, A. Carpinteri, Fractals and Fractional Calculus in Continuum Mechanics (Springer, New York, 1997) 
[9] V.E. Tarasov, "Continuous medium model for fractal media" Phys. Lett. A 336 (2005) 167-174; "Fractional hydrodynamic equations for fractal media" Ann. Phys. 318 (2005) 286-307; "Fractional Fokker-Planck equation for fractal media" Chaos 15 (2005) 023102; "Dynamics of fractal solid" Int. J. Mod. Phys. B 19 (2005) 4103-4114; "Wave equation for fractal solid string" Mod. Phys. Lett. B 19 (2005) 721-728; "Possible experimental test of continuous medium model for fractal media" Phys. Lett. A 341 (2005) 467-472; "Gravitational field of fractal distribution of particles" Celes. Mech. Dynam. Astron. 19 (2006) 1-15; "Multipole moments of fractal distribution of charges" Mod. Phys. Lett. B 19 (2005) 1107-1118; "Electromagnetic field of fractal distribution of charged particles" Physics of Plasmas 12 (2005) 082106; "Magnetohydrodynamics of fractal media" Physics of Plasmas 13 (2006) 052107; "Electromagnetic fields on fractals" Modern Physics Letters A 21 (2006) 1587-1600.

[10] V.E. Tarasov, "Fractional generalization of Liouville equation" Chaos 14 (2004) 123-127; "Fractional systems and fractional Bogoliubov hierarchy equations" Phys. Rev. E 71 (2005) 011102; "Fractional Liouville and BBGKI equations" J. Phys. Conf. Ser. 7 (2005) 1733; "Transport equations from Liouville equations for fractional systems" Int. J. Mod. Phys. B. 20 (2006) 341-354; "Fractional generalization of gradient systems" Lett. Math. Phys. 73 (2005) 49-58; "Fractional generalization of gradient and Hamiltonian systems" J. Phys. A 38 (2005) 5929-5943; "Fractional variations for dynamical systems: Hamilton and Lagrange approaches" J. Phys. A 39 (2006) 8409-8425.

[11] J. Klafter, A. Blumen, M.F. Shlesinger "Stochastic pathways to anomalous diffusion" Phys. Rev. A 35 (1987) 3081-3085.

[12] A.I. Saichev, G.M. Zaslavsky, "Fractional kinetic equations: solutions and applications" Chaos 7 (1997) 753-764.

[13] R. Gorenflo, F. Mainardi, "Fractional diffusion processes: Probability distribution and continuous time random walk. Lecture Notes in Physics" 621 (2003) 148-166.

[14] R. Metzler, J. Klafter, "The restaurant at the end of the random walk: recent developments in the description of anomalous transport by fractional dynamics" J. Phys. A 37 (2004) R161-R208; "The random walk's guide to anomalous diffusion: A fractional dynamics approach" Phys. Rep. 339 (2000) 1-77.

[15] V.V. Uchaikin, "Self-similar anomalous diffusion and Levy-stable laws" Physics-Uspekhi 46 (2003) 821-849; "Anomalous diffusion and fractional stable distributions" J. Exper. Theor. Phys. 97 (2003) 810-825.

[16] N. Laskin, G.M. Zaslavsky, "Nonlinear fractional dynamics on a lattice with long-range interactions" Physica A 368 (2006) 38-54.

[17] V.E. Tarasov, G.M. Zaslavsky, "Fractional dynamics of coupled oscillators with long-range interaction" Chaos 16 (2006) 023110; "Fractional dynamics of systems with long-range interaction" Commun. Nonlin. Sci. Numer. Simul. 11 (2006) 885-898.

[18] N. Korabel, G.M. Zaslavsky, V.E. Tarasov, "Coupled oscillators with power-law interaction and their fractional dynamics analogues" Commun. Nonlin. Sci. Numer. Simul. 12 (2007) 1405-1417, (math-ph/0603074) 
[19] N. Korabel, G.M. Zaslavsky "Transition to chaos in discrete nonlinear Schrödinger equation with long-range interaction" Physica A 378 (2007) 223-237, (math-ph/0607030).

[20] F.J. Dyson, "Existence of a phase-transition in a one-dimensional Ising ferromagnet" Commun. Math. Phys. 12 (1969) 91-107; "Non-existence of spontaneous magnetization in a one-dimensional Ising ferromagnet" Commun. Math. Phys. 12 (1969) 212-215; "An Ising ferromagnet with discontinuous long-range order" Commun. Math. Phys. 21 (1971) 269283.

[21] G.S. Joyce, "Absence of ferromagnetism or antiferromagnetism in the isotropic Heisenberg model with long-range interactions" J. Phys. C: Solid State Phys. 2 (1969) 1531-1533.

[22] J. Frohlich, R. Israel, E.H. Lieb, B. Simon, "Phase transitions and reflection positivity I. General theory and long-range lattice model" Commum. Math. Phys. 62 (1978) 1-34.

[23] H. Nakano, M. Takahashi, "Quantum Heisenberg chain with long-range ferromagnetic interactions at low temperatures" J. Phys. Soc. Japan 63 (1994) 926-933; "Quantum Heisenberg model with long-range ferromagnetic interactions" Phys. Rev. B 50 (1994) 10331-10334; "Magnetic properties of quantum Heisenberg ferromagnets with long-range interactions" Phys. Rev. B 52 (1995) 6606-6610.

[24] J.R. Sousa, "Phase diagram in the quantum XY model with long-range interactions" Eur. Phys. J. B 43 (2005) 93-96.

[25] S. Shima, Y. Kuramoto, "Rotating spiral waves with phase-randomized core in nonlocally coupled oscillators" Phys. Rev. E 69 (2004) 036213.

[26] D. Mukamel, S. Ruffo, N. Schreiber, "Breaking of ergodicity and long relaxation times in systems with long-range interactions" Phys. Rev. Lett. 95 (2005) 240604; J. Barre, F. Bouchet, T. Dauxois, S. Ruffo, "Large deviation techniques applied to systems with long-range interactions" J. Stat. Phys. 119 (2005) 677-713.

[27] O.M. Braun, Y.S. Kivshar, "Nonlinear dynamics of the Frenkel-Kontorova model" Phys. Rep. 306 (1998) 2-108.

[28] Y. Ishimori, "Solitons in a one-dimensional Lennard-Jones lattice" Prog. Theor. Phys. 68 (1982) 402-410.

[29] O.M. Braun, Y.S. Kivshar, I.I. Zelenskaya, "Kinks in the Frenkel-Kontorova model with long-range interparticle interactions" Phys. Rev. B 41 (1990) 7118-7138.

[30] Yu.B. Gaididei, S.F. Mingaleev, P.L. Christiansen, K.O. Rasmussen, "Effects of nonlocal dispersive interactions on self-trapping excitations" Phys. Rev. E 55 (1997) 6141-6150; S.F. Mingaleev, Y.B. Gaididei, F.G. Mertens, "Solitons in anharmonic chains with powerlaw long-range interactions" Phys. Rev. E 58 (1998) 3833-3842; S.F. Mingaleev, Y.B. Gaididei, F.G. Mertens, "Solitons in anharmonic chains with ultra-long-range interatomic interactions" Phys. Rev. E 61 (2000) R1044-R1047; Yu. Gaididei, N. Flytzanis, A. Neuper, F.G. Mertens, "Effect of nonlocal interactions on soliton dynamics in anharmonic lattices" Phys. Rev. Lett. 75 (1995) 2240-2243; K.O. Rasmussen, P.L. Christiansen, M. Johansson, Yu.B. Gaididei, S.F. Mingaleev, "Localized excitations in discrete nonlinear Schroedinger systems: Effects of nonlocal dispersive interactions and noise" Physica D, 113 (1998) 134-151. 
[31] V.L. Pokrovsky, A. Virosztek, "Long-range interactions in commensurate-incommensurate phase transition" J. Phys. C 16 (1983) 4513-4525.

[32] S. Flach, "Breathers on lattices with long-range interaction" Phys. Rev. E 58 (1998) R4116-R4119.

[33] A.V. Gorbach, S. Flach, "Compactlike discrete breathers in systems with nonlinear and nonlocal dispersive terms" Phys. Rev. E 72 (2005) 056607.

[34] P. Woafo, T.C. Kofane, A.S. Bokosah, "Kink static properties in a discrete $\varphi^{4}$ chain with long-range interactions" Phys. Rev. B 48 (1993) 10153-10159; J.C. Cressoni, M.L. Lyra, "The nature of electronic states in a disordered chain with long-ranged hopping amplitudes" Physica A 256 (1998) 18-29; R.P.A. Lima, M.L. Lyra, J.C. Cressoni, "Multifractality of one electron eigen states in 1D disordered long-range models" Physica A 295 (2001) 154-157.

[35] G.L. Alfimov, V.M. Eleonsky, L.M. Lerman, "Solitary wave solutions of nonlocal sineGordon equations" Chaos 8 (1998) 257-271.

[36] G.L. Alfimov, V.G. Korolev, "On multikink states described by the nonlocal sine-Gordon equation" Phys. Lett. A 246 (1998) 429-435.

[37] G. Alfimov, T. Pierantozzi, L. Vazquez, "Numerical study of a fractional sine-Gordon equation" in: Fractional differentiation and its applications, A. Le Mehaute, J.A. Tenreiro Machado, L.C. Trigeassou, J. Sabatier (Eds.), Proceedings of the IFAC-FDA'04 Workshop, Bordeaux, France, July 2004; pp. 153-162.

[38] A.I. Saichev, G.M. Zaslavsky, "Fractional kinetic equations: solutions and applications" Chaos 7 (1997) 753-764.

[39] F. Mainardi, Yu. Luchko, G. Pagnini, "The fundamental solution of the space-time fractional diffusion equation" Fract. Calc. Appl. Anal. 4 (2001) 153-192.

[40] H. Weitzner, G.M. Zaslavsky, "Some applications of fractional derivatives" Commun. Nonlin. Sci. Numer. Simul. 8 (2003) 273-281.

[41] V.E. Tarasov, G.M. Zaslavsky, "Fractional Ginzburg-Landau equation for fractal media" Physica A 354 (2005) 249-261.

[42] V.E. Tarasov, G.M. Zaslavsky, "Dynamics with low-level fractionality" Physica A 368 (2006) 399-415.

[43] A.V. Milovanov, J.J. Rasmussen, "Fractional generalization of the Ginzburg-Landau equation: an unconventional approach to critical phenomena in complex media" Phys. Lett. A 337 (2005) 75-80.

[44] V.E. Tarasov, "Psi-series solution of fractional Ginzburg-Landau equation" J. Physics A 39 (2006) 8395-8407.

[45] A. Erdèlyi, W. Magnus, F. Oberhettinger, F.G. Tricomi, Higher Transcendental Functions, Vol. 1. (Krieger, New York, 1981) pp.30-31.

[46] H. Bateman, A. Erdelyi, Higher Transcendental Functions Vol.1. (Mc Graw-Hill, New York, 1953) Sec. 1.13. 
[47] A.P. Prudnikov, Yu.A. Brychkov, O.I. Marichev, Integrals and Series, Vol. 1: Elementary Functions (Gordon and Breach, New York, 1986)

[48] J. Burgers, The Nonlinear Diffusion Equation (Reidel, Dordrecht, Amsterdam, 1974)

[49] P. Biler, T. Funaki, W.A. Woyczynski, "Fractal Burger equation" J. Differ. Eq. 148 (1998) 9-46.

[50] S. Momani, "An explicit and numerical solutions of the fractional KdV equation" Math. Comput. Simul. 70 (2005) 110-1118.

[51] P. Miskinis, "Weakly nonlocal supersymmetric KdV hierarchy" Nonlinear Analysis: Modelling and Control 10 (2005) 343-348.

[52] M.M. Meerschaert, J. Mortensen, S.W. Wheatcraft, "Fractional vector calculus for fractional advection-dispersion" Physica A 367 (2006) 181-190.

[53] R. Schumer, D.A. Benson, M.M. Meerschaert, S. W. Wheatcraft, "Eulerian derivation of the fractional advection-dispersion equation" Journal of Contaminant Hydrology, 48 (2001) 6988. 


\section{Appendix}

\begin{tabular}{|c|c|}
\hline$J(n)$ & $\hat{J}_{\alpha}(k)-\hat{J}_{\alpha}(0)$ \\
\hline$\left(\frac{(-1)^{n} \pi^{\alpha+1}}{\alpha+1}-\frac{(-1)^{n} \pi^{1 / 2}}{(\alpha+1)|n|^{\alpha+1 / 2}} L_{1}(\alpha+3 / 2,1 / 2, \pi n)\right)$ & $|k|^{\alpha}$ \\
\hline$\frac{(-1)^{n}}{n^{2}}$ & $(1 / 2) k^{2}$ \\
\hline$\frac{1}{n^{2}}$ & $\frac{1}{2}\left[k^{2}-2 \pi k\right], \quad(0 \leq k \leq 2 \pi)$ \\
\hline$|n|^{-(\beta+1)}, \quad(0<\beta<2, \beta \neq 1)$ & $2 \Gamma(-\beta) \cos (\pi \beta / 2)|k|^{\beta}$ \\
\hline$|n|^{-(\beta+1)}, \quad(\beta>2, \beta \neq 3,4, \ldots)$ & $-\zeta(\alpha-1) k^{2}$ \\
\hline$\frac{(-1)^{n}}{\Gamma(1+\alpha / 2+n) \Gamma(1+\alpha / 2-n)} \quad(\beta>-1 / 2)$ & $\frac{2^{\alpha}}{\Gamma(\alpha+1)} \sin ^{\alpha}\left(\frac{k}{2}\right)$ \\
\hline$\frac{(-1)^{n}}{a^{2}-n^{2}}$ & $\frac{\pi}{a \sin (\pi a)} \cos (a k)-\frac{1}{a^{2}} \quad(0<k<2 \pi)$ \\
\hline$J(n)=1 / n !$ & $e^{\cos k} \cos (\sin k)$ \\
\hline
\end{tabular}

